\title{
Evidence for Hypothalamus-Pituitary-Adrenal Axis and Immune Alterations at Prodrome of Psychosis in Males
}

\author{
Evangelos Karanikas ${ }^{1,2} \bowtie$, Evangelos Ntouros ${ }^{2}$, Dimitrios Oikonomou ${ }^{2}$, \\ Georgios Floros 3 , loannis Griveas ${ }^{4}$, and Georgios Garyfallos ${ }^{3}$ \\ ${ }^{1}$ School of Medicine, Rural Clinical School, The University of Queensland, Toowoomab, Australia \\ ${ }^{2}$ Psychiatric Department, General Military Hospital of Thessaloniki, Thessaloniki, Greece \\ ${ }^{3}$ 2nd Psychiatric Department, Aristotle University of Thessaloniki, Psychiatric Hospital of Thessaloniki, Thessaloniki, Greece \\ ${ }^{4}$ General Military Hospital of Athens, Athens, Greece
}

We aimed to investigate the inflammatory substrate in psychosis by evaluating both the Hypothalamus-Pituitary-Adrenal axis function and immune state at prodrome. This involved the recruitment of Ultra High Risk (UHR) of Psychosis subjects, Healthy Controls (HC) and patients with established Schizophrenia (CHRON). Serum cortisol at 3 different times throughout the day was measured. The Dexamethasone Suppression Test was performed plus 12 circulating cytokines were measured. The UHR subjects presented increased IL-4 levels compared with both the $\mathrm{HC}$ and $\mathrm{CHRON}$ patients. In contrast the UHR differed only from the CHRON group regarding the endocrine parameters. In conclusion, IL-4 appears to play a key role at prodrome.

Psychiatry Investig 2017;14(5):703-707

Key Words Cortisol, Cytokines, Inflammation, Prodrome, Ultra high risk.

\section{INTRODUCTION}

Inflammation as an underlying pathophysiological substrate of psychosis has recently been intensely investigated. Evidence for up regulation of Hypothalamo-Pituitary-Adrenal (HPA) Axis function at First Episode of Psychosis has been shown. ${ }^{1}$ Preliminary evidence favoring increase of circulating cortisol appears to be present even from the prodromal stage. ${ }^{2}$ Similarly, findings of immunological parameters in cohorts with psychosis increasingly hint at dysregulation of the immune system with cytokines constituting a promising field for investigation, yet the prodromal stage still remains minimally investigated. ${ }^{3}$ Thus, we aimed to test the hypothesis of immune and endocrine dysregulation at prodrome by measuring circulating cytokines representative of different arms

Received: July 15, 2016 Revised: September 11, 2016

Accepted: October 13, 2016 Available online: June 16, 2017

$\triangle$ Correspondence: Evangelos Karanikas, MD, PhD

School of Medicine, Rural Clinical School, The University of Queensland, 152 West St, QLD 4350, Toowoomba, Australia

Psychiatric Department, General Military Hospital of Thessaloniki, 424 Thessaloniki Ring Road, 56429 Efkarpia, Thessaloniki, Greece

Tel: +30 2310 224490, Fax: +30 2310 381010, E-mail: epkarani@yahoo.com

(c) This is an Open Access article distributed under the terms of the Creative Commons Attribution Non-Commercial License (http://creativecommons.org/licenses/bync/4.0) which permits unrestricted non-commercial use, distribution, and reproduction in any medium, provided the original work is properly cited. of immunity; namely the innate immunity (IL-1, IL-6, TNF-a), the Thelper-1 (Th-1) (IFN- $\gamma$, IL-2, IL-8, IL-12p70, TNF-b) and the Th-2 ( IL-4, IL-5, IL-6, IL-10) arms of the adaptive immunity and the newly discovered Th-17 immune response (IL-17A) as well as components of the HPA axis function in Ultra High Risk (UHR) of Psychosis subjects and comparing them with Healthy Controls (HC) and patients with established Schizophrenia (CHRON).

\section{METHODS}

The study was conducted, from May 2012 up until May 2014. The recruitment of the UHR group, involved 12 male subjects having been referred for further evaluation of their deterioration in their behavior/function and the Personal Assessment and Crisis Evaluation criteria were applied using the Comprehensive Assessment of At Risk Mental States. ${ }^{4}$ The CHRON group consisted of 16 male patients with established diagnosis of Schizophrenia, which was confirmed with the Structured Clinical Interview for DSM-IV-TR Disorders (SCID) for DSMIV-TR, in relapse state. Finally we included 23 male participants as HC; the psychiatric morbidity was excluded through SCID-NonPatient and no one had first degree relatives diagnosed with psychosis. 
The symptom severity in both UHR and CHRON groups was assessed with the Greek version of the Positive and Negative Syndrome Scale (PANSS) and its subscales, ${ }^{5}$ Positive Factor (PANSS PF), Negative Factor (PANSS NF), General Psychopathology (PANSS GP) and Total score (PANSS TOTAL).

To be included in the study, the CHRON patients were antipsychotic medication free for at least 15 days prior to their admission and did not receive any medication for more than 3 days prior to the blood sampling. The UHR were medication naïve.

All the participants were physically healthy with no signs of active inflammation for at least 15 days prior to the study. Any illicit drug users were excluded.

All participants gave informed consent. The local ethical scientific committees of the involved hospitals approved the study protocol. The study was performed in accordance with the latest edition of the Declaration of Helsinki.

The day 1 of blood sampling, samples were collected at 3 separate points in time; 08:00, 12:00 and 18:00. At 23:00 of day 1,1 mg of Dexamethasone (Dex), was given. At 08:00 of day 2, post/Dex, another blood sample was collected.

Cortisol was measured from the 3 samples at day 1 and the post-Dex sample at day 2 , with radioimmunoassay method.

The cytokines were measured on day 1 at 08:00 to avoid any effect of Dex. The cytokine evaluation involved the principles of Fluorescent Bead Immunoassay and FlowCytomix.

\section{Statistical analysis}

Statistical analyses were performed using SPSS 20 software (IBM Corp., Armonk, NY, USA). All cytokine values were logarithmically transformed. Multi-group differences were assessed by way of the Kruskal-Wallis test, individual group differences were assessed by way of Mann-Whitney tests with the $\mathrm{p}$ value corrected for multiple comparisons using the Bonferroni correction, target $\mathrm{p}$ set at $<0.016$ for an alpha of $<0.05$. Intra-group correlations were assessed with the Spearman rs statistic. The cortisol values were assessed with the formulas of 'Area Under Curve (AUC) with respect to increase' (AUCi) and 'AUC with respect to ground' (AUCg). We computed the absolute difference between cortisol values pre- and post- administering Dex (pre-post/Dex cort). We also compared the rate of cortisol suppressors of each group depending on whether they showed post/Dex cort values below the cutoff $5 \mu \mathrm{g} / \mathrm{dL}$.

\section{RESULTS}

Group demographics and clinical data are presented in Table 1. Body mass index (BMI), smoking and antipsychotic treatment did not correlate significantly either with cortisol AUCg or with cytokine levels (all p $>0.05$ ), and were thus not controlled

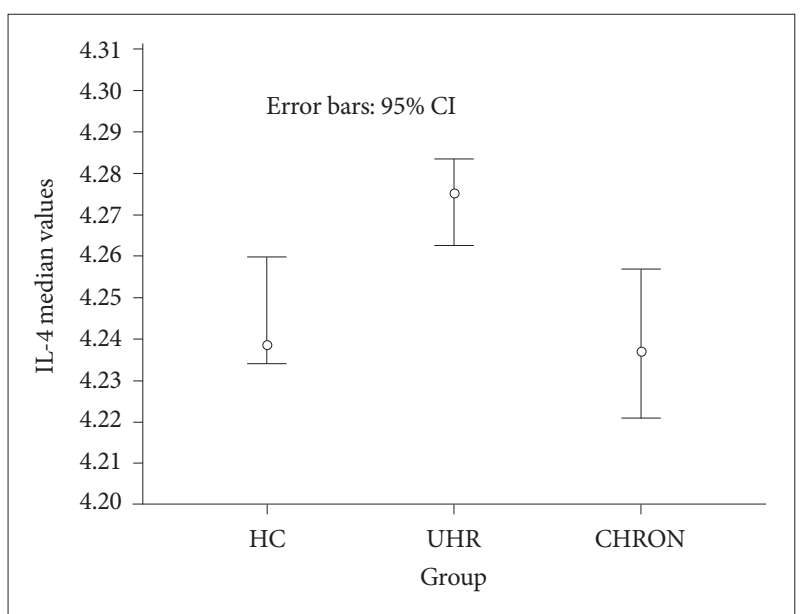

Figure 1. IL-4 median values with $95 \% \mathrm{Cl}$. UHR: ultra high risk, $\mathrm{CHRON}$ : chronic patients with schizophrenia, $\mathrm{HC}$ : healthy controls.

as confounders. Age correlated with IL-4 ( $r s=-0.25, \mathrm{p}=0.04)$ and cortisol AUCg (rs=-0.378, $\mathrm{p}=0.006)$ and the effects of potential confounding were thus considered in the analysis of findings pertaining to the $\mathrm{CHRON}$ group, since they were significantly older $(\mathrm{p}<0.001)$.

In regards to the cytokines concentrations, the UHR group presented significantly increased IL-4 compared with both the HC ( $p=0.01$ ) and CHRON ( $p=0.001)$ groups (Figure 1). For the comparison against the $\mathrm{CHRON}$ group, when the age confounder was included in the analysis the difference in IL-4 levels compared to UHR was no longer significant ( $p>0.05)$.

Regarding the group comparisons for cortisol levels, the mean $\pm S D$ values for AUCg, AUCi, pre-post/Dex cort and the $\mathrm{p}$ values are shown in Table 1 . The AUCi values were negative indicating the cortisol decline during the day. For the comparison against the $\mathrm{CHRON}$ group, when the age confounder was included in the analysis of covariance, the difference in cortisol AUCg compared to UHR was no longer significant. One subject from the CHRON group suppressed the post/Dex cort below the cutoff $(5 \mu \mathrm{g} / \mathrm{dL})$ as opposed to none from each of the UHR and HC groups.

\section{DISCUSSION}

This study is the first to have evaluated parameters from both the HPA axis and the immune system in a cohort at prodrome and compared them with Schizophrenia patients and HC. The findings suggest that the UHR group presented significantly increased serum IL-4 levels compared with both the $\mathrm{HC}$ and CHRON groups. Regarding the HPA axis function, the findings were more subtle as there was no clear deviation from the HC. Specifically, the UHR group showed significantly higher cortisol AUCg only in comparison with the CHRON group. Moreover the pre-post/Dex cort and the cortisol sup- 
pression rate of DST in the UHR subjects did not deviate from the HC counterparts in either of them.

Our finding of a trend for higher cortisol AUCg in the UHR compared with $\mathrm{HC}$ may be suggestive of a trend for increased baseline cortisol secretion at prodrome. This finding, despite not succeeding significant deviation from the HC-possibly due to the small UHR cohort-, could be interpreted as standing in line with the rest of other preliminary findings. ${ }^{2}$

Moreover, the UHR group in our study, exhibited evidence for heightened cortisol AUCg and AUCi change/decline (at trend level) compared with the CHRON group, whereas the evidence was less clear when compared with the HC. The hitherto few studies, comparing HPA axis function among prodromal subjects, $\mathrm{HC}$ and patients with Schizophrenia, involved At Genetic Risk of Psychosis (AGRP) subjects and suggested higher cortisol secretion for the Schizophrenia groups. ${ }^{6,7}$ An AGRP cohort, as opposed to our UHR sample, is less likely to convert to full psychosis, thus providing a possible explanation why the cortisol levels of the UHR group were increased compared to the $\mathrm{CHRON}$ group. An alternative interpretation may relate to the confounding effect of age.

Regarding the DST results of the present study, the evidence is inconclusive. The pre-post/Dex cort levels in the UHR did not differ from the HC; plus no non suppressor UHR subject

Table 1. Group demographics, clinical data, cytokine and cortisol levels

\begin{tabular}{|c|c|c|c|c|}
\hline & $\mathrm{HC}(\mathrm{N}=23)$ & $\operatorname{UHR}(\mathrm{N}=12)$ & CHRON $(\mathrm{N}=16)$ & Group comparison \\
\hline Demographics & & & & $\mathrm{p}$ \\
\hline Age & $27.04(2.9)$ & $24.5(3.1)$ & $35.56(5.5)$ & $<0.001(\mathrm{a}, \mathrm{c})$ \\
\hline Education & $13.26(1.9)$ & $13.16(2.1)$ & $12.18(1.9)$ & 0.232 \\
\hline BMI & $24.52(2.1)$ & $24.15(1.9)$ & $29.19(2.2)$ & $<0.001(\mathrm{a}, \mathrm{c})$ \\
\hline Smoking (yes\no) & $7 / 16$ & $7 / 5$ & $13 / 3$ & 0.007 \\
\hline Antipsychotic treatment & & & $476.43(187.4)$ & \\
\hline Clinical assesment & & & & $\mathrm{p}$ \\
\hline PANSS PF & & $4.92(9.06)$ & $17.31(5.9)$ & 0.002 \\
\hline PANSS NF & & $5.33(9.78)$ & $20.93(7.6)$ & 0.001 \\
\hline PANSS GP & & $8.92(16.27)$ & $35.25(9.1)$ & $<0.001$ \\
\hline PANSS total & & $19.17(34.75)$ & $73.5(19.8)$ & 0.001 \\
\hline Cytokines & & & & $\mathrm{p}$ \\
\hline IL-1b & $68(44,82)$ & $64(40,67)$ & $57(50,75)$ & 0.55 \\
\hline IL-2 & $97(93,101)$ & $95(94,97)$ & $92(91,97)$ & 0.75 \\
\hline IL-4 & $68(68,70)$ & $71(70,71)$ & $68(67,69)$ & $0.001\left(\mathrm{a}^{\ddagger}\right), 0.01\left(\mathrm{~b}^{\ddagger}\right)$ \\
\hline IL-5 & $3.4(0.4,45.9)$ & $0.4(0.4,3.8)$ & $3.5(0.4,81.2)$ & 0.256 \\
\hline IL-8 & $381(125,1168)$ & $331(0.2,1028)$ & $232(19,544)$ & 0.554 \\
\hline IL-10 & $10(3,36)$ & $6(1,7)$ & $6(1,20)$ & 0.281 \\
\hline IL-12p70 & $12(11,17)$ & $11(10,12)$ & $10(9,15)$ & 0.318 \\
\hline IFN- $\gamma$ & $22(16,36)$ & $20(17,27)$ & $21(18,27)$ & 0.859 \\
\hline TNF-a & $0.8(0.8,4)$ & $0.8(0.8,0.8)$ & $0.8(0.8,0.8)$ & 0.42 \\
\hline TNF-b & $0.6(0.6,0.6)$ & $0.6(0.6,0.6)$ & $0.6(0.6,0.6)$ & 0.185 \\
\hline Cortisol & & & & $\mathrm{p}$ \\
\hline AUCg & $165.33(54.56)$ & $206.4(72.85)$ & $155.74(53.24)$ & $0.047\left(\mathrm{a}^{*}\right), 0.074\left(\mathrm{~b}^{\dagger}\right)$ \\
\hline AUCi & $-59.78(38.46)$ & $-80.63(56.45)$ & $-35.33(37.15)$ & $0.082\left(\mathrm{a}^{\dagger}\right)$ \\
\hline Pre-post/Dexcort & $11.3(2.19)$ & $12.80(3.49)$ & $9.1(2.09)$ & $0.045\left(\mathrm{a}^{*}\right)$ \\
\hline
\end{tabular}

IL-6 and IL-17A levels were under detection limits in $>50 \%$ cases. ${ }^{*} \mathrm{p}<0.05,{ }^{\dagger} \mathrm{p}>0.05 \& \mathrm{p}<0.1,{ }^{\ddagger}$ Cytokines Mann-Whitney tests with the $\mathrm{p}$ value corrected for multiple comparisons using the Bonferroni correction (target $p$ set at $<0.016$ for an alpha of $<0.05$ ). UHR: At Clinical Risk for Psychosis, FEP: First Episode Psychosis, CHRON: Chronic with Schizophrenia; Age, Education in years, BMI: body mass index; Antipsychotic treatment: CPZ equivalents in mg/day, PANSS: Positive and Negative Syndrome Scale, PF: Positive Factor, NF: Negative Factor, GP: General Psychopathology, TOTAL: total score, IL: interleukin, IFN: interferon, TNF: tumor necrosis factor, AUCg: Area Under curve with respect to ground, AUCi: Area Under Curve with respect increase, pre-post/Dex cort: cortisol levels prior Dexamethasone-cortisol post Dex Variables presented in mean (SD), only cytokines in median (25\%, $75 \%$ percentile). The cytokines levels in pg/mL, AUCg-AUGi in $\mu \mathrm{g} / \mathrm{dL} / \mathrm{min}$, pre-post Dex cort in $\mu \mathrm{g} / \mathrm{dL}$. Cortisol p: Between -group differences with Mann Whitney U test p set at 0.05 a: UHR vs. CHRON, b: UHR vs. $\mathrm{HC}, \mathrm{c}: \mathrm{CHRON}$ vs. HC 
was detected, thus indicating no alteration to the HPA axis suppression capacity. This in turn could suggest no significant deviation of the glucocorticoid receptors' negative feedback action and/or number in the prodromal group.

Regarding the immune domain, IL-4 was the only cytokine to have been gauged significantly increased, in the UHR group, compared with both the $\mathrm{HC}$ and $\mathrm{CHRON}$. This finding may suggest that IL-4 can have immunoprotective effect which converges with its role in activation of Th-2 immunity and the subsequent anti-inflammatory action. Thus, an increased mobilization of IL-4 in UHR, offering a last barrier of resistance to a coming full blown psychotic episode, could be speculated. This interpretation can be further substantiated by the positive association between IL-4 and AUCg cortisol, exhibited only by the UHR group (data not shown), and the knowledge that cortisol triggers Th-2 immune response.

Conversely viewing, the heightened IL-4 in the UHR, could imply a predisposition of the UHR group towards the impending frank psychosis. In other words, IL-4 might not have increased on a compensatory neuroprotective basis but rather etiologically. In this case, the hypothesis of a Th-2 shift in psychosis could be substantiated. ${ }^{8}$ In line with this presumption stand preliminary findings from UHR studies, suggesting a role for IL-6, IL-7 and IL-8. ${ }^{9,10}$ These cytokines have been hypothesized that participate in the orchestration of the autoimmune response. ${ }^{11,12}$

Regarding the CHRON group in our study, interestingly, both their HPA axis function components and immune profile did not differ from the HC. The findings are not as surprising, should someone take into account the highly divergent findings of a recent systematic review, ${ }^{13}$ incorporating 104 studies of HPA axis function in mostly chronic patients with Schizophrenia. The diversity was attributed to the impact of comorbidity, phase of illness, presented symptoms, medication, setting and trauma which along with factors such as age, BMI, tobacco smoking, cannabis, night shift work, exert their effect on the HPA axis function. ${ }^{14-16}$ Similarly, from the immune point of view, the non-deviant cytokines levels of our CHRON subjects compared with the HC, oppose previous studies, where a role for IL-6, IL-2, IL-1, IL-10, IFN- $\gamma$ and TNF-a in chronicity was suggested. ${ }^{17-19}$ Yet, our findings are in line with other studies ${ }^{20,21}$ failing to find any deviation. Notably, the CHRON group of the present study was not age, BMI, smoking status matched with the HC group. These demographic parameters are known to affect circulating cytokines' levels ${ }^{22}$ and along with chronicity and previous exposure to medication may account for the CHRON's non-deviant immune profile compared with the HC (although the studied cytokines were not significantly correlated with the pre-mentioned parameters-with age exemption).
In conclusion, this study constitutes a preliminary approach to investigate the endocrine and immune function at prodrome. In the UHR group, IL-4 levels deviated from the HC, thus suggesting its key role during this stage. In contrast the evidence for alterations in the HPA axis function was less clear. Larger longitudinal studies would provide further insight in the etiological sequence and the extent that inflammation relates to psychosis.

\section{Acknowledgments}

The Authors wish to thank Associate Professor Dr Papa, Anna Director of the Department of Immunology and AIDS Report Centre, Aristotle University of Thessaloniki, Greece, and Dr. Gerou, Spyridon Director of the Branch of Immunology/ "ANALYSIS" Laboratory of Microbiology and Biochemisty, Thessaloniki, Greece, affiliated with Aristotle University of Thessaloniki, Greece for their contribution in the study.

\section{REFERENCES}

1. Karanikas E, Antoniadis D, Garyfallos G. The role of cortisol in first episode of psychosis: a systematic review. Curr Psychiatry Rep 2014;16: 503-513.

2. Karanikas E, Garyfallos G. Role of cortisol in patients at risk for psychosis mental state and psychopathological correlates: a systematic review. Psychiatry Clin Neurosci 2015;69:268-282.

3. Miller BJ, Buckley P, Seabolt W, Mellor A, Kirkpatrick B. Meta-analysis of cytokine alterations in schizophrenia: clinical status and antipsychotic effects. Biol Psychiatry 2011;70:663-671.

4. Yung AR, Yuen HP, Mc Gorry PD, Phillips LJ, Kelly D, Dell'Olio M, et al. Mapping the onset of psychosis: the Comprehensive Assessment of At-Risk Mental States. Aust N Z J Psychiatry 2005;39:964-971.

5. Lykouras E, Botsis A, Oulis P. The Positive and Negative Syndrome Scale (PANSS) [in Greek]. Athens, Greece: Tsiveriotis Ed; 1994.

6. Spelman LM, Walsh P, Sharifi N, Collins P, Thakore JH. Impaired glucose tolerance in first-episode drug-naïve patients with schizophrenia. Diabet Med 2007;24:481-485.

7. Yildirim O, Dogan O, Semiz M, Kilicli F. Serum cortisol and dehydroepiandrosterone-sulfate levels in schizophrenic patients and their firstdegree relatives. Psychiatry Clin Neurosci 2011;65:584-591.

8. Muller N, Schwarz MJ. Immune system and schizophrenia. Curr Immunol Rev 2010;6:213-220.

9. Stojanovic A, Martorell L, Montalvo I, Ortega L, Monseny R, Vilella E, et al. Increased serum interleukin-6 levels in early stages of psychosis: associations with at-risk mental states and the severity of psychotic symptoms. Psychoneuroendocrinology 2014;41:23-32.

10. Perkins DO, Jeffries CD, Addington J, Bearden CE, Cadenhead KS, Cannon TD, et al. Towards a psychosis risk blood diagnostic for persons experiencing high-risk symptoms: preliminary results from the NAPLS project. Schizophr Bull 2015;41:419-428.

11. Zhang XY, Zhou DF, Zhang PY, Wu GY, Cao LY, Shen YC. Elevated interleukin-2, interleukin-6 and interleukin-8 serum levels in neuroleptic-free schizophrenia: association with psychopathology. Schizophr Res 2002;57:247-258.

12. Dooms H. Interleukin-7: fuel for the autoimmune attack. J Autoimmun 2013;45:40-48.

13. Bradley AJ, Dinan TG. Review: a systematic review of hypothalamicpituitary-adrenal axis function in schizophrenia: implications for mortality. J Psychopharmacol 2010;24(4 Suppl):91-118.

14. Bose M, Olivan B, Laferrere B. Stress and obesity: the role of the hypothalamic pituitary-adrenal axis in metabolic disease. Curr Opin Endocrinol Diabetes Obes 2009;16:340-346.

15. de Leon J, Diaz FJ. A meta-analysis of worldwide studies demonstrates 
an association between schizophrenia and tobacco smoking behaviors. Schizophr Res 2005;76:135-157.

16. Di Forti M, Morrison Pd, Butt A, Murray Rm. Cannabis use and psychiatric and cognitive disorders: the chicken or the egg? Curr Opin Psychiatry 2007;20:228-234.

17. Lin A, Kenis G, Bignotti S, Tura GJ, De Jong R, Bosmans E, et al. The inflammatory response system in treatment resistant schizophrenia: increased serum interleukin-6. Schizophr Res 1998;32:9-15.

18. Sirota P, Schild K, Elizur A, Djaldetti M, Fishman P. Increased interleukin-1 and interleukin-3 like activity in schizophrenic patients. Prog Neuropsychopharmacol Biol Psychiatry 1995;19:75-83.

19. Kaminska T, Wysocka A, Marmurowska-Michalowska H, DubasSlemp H, Kandefer-Szerszen M. Investigation of serum cytokine levels and cytokine production in whole blood cultures of paranoid schizo- phrenic patients. Arch Immunol Ther Exp (Warsz) 2001;49:439-445.

20. Kim YK, Myint AM, Verkerk R, Scharpe S, Steinbusch H, Leonard B. Cytokine changes and tryptophan metabolites in medication naïve and medication-free schizophrenic patients. Neuropsychobiology 2009; 59:123-129.

21. Maes M, Bosmans E, Ranjan R, Vandoolaeghe E, Meltzer HY, De Ley M, et al. Lower plasma CC16, a natural anti-inflammatory protein, and increased plasma interleukin-1 receptor antagonist in schizophrenia: effects of antipsychotic drugs. Schizophr Res 1996;21:39-50.

22. O'Connor MF, Bower JE, Cho HJ, Creswell JD, Dimitrov S, Hamby ME, et al. To assess, to control, to exclude: effects of biobehavioral factors on circulating inflammatory markers. Brain Behav Immun 2009;23:887897. 